\title{
Espèces nouvelles ou mal connues de Cholevidae (Coleoptera)
}

\author{
Michel PERREAU \\ Laboratoire de Magnétisme des surfaces, Université Paris 7, \\ 2, place Jussieu, F-75251 Paris cedex 05, France.
}

New or poorly known species of Cholevidae (Coleoptera). - New data are given on the morphology and the distribution of Choleva gabriellae Giachino, and Attaephilus scheuerni Perreau. The descriptions of the following new species are given: Ptomaphaginus balazuci n. sp., Ptomaphaginus baliensis n. sp., Ptomaphaginus riedeli n. sp., and Choleva (Protocatops) medvedevi n. subgen., n. sp. With the latter species the distribution of Choleva is extended to Vietnam. Some apomorphic characters shared with Choleva henroti Szymczakowski, lead to the description of a new subgenus: Protocatops.

Key-words: Coleoptera - Cholevidae - Taxonomy - Distribution.

\section{INTRODUCTION}

Nous regroupons dans cet article un certain nombre d'observations sur des Cholevidae originaires de la région méditerranéenne et de l'Asie du sud-est. L'aire de répartition de certaines espèces se trouve précisée, c'est le cas pour Choleva gabriellae Giachino, dont seul le mâle était jusqu'à présent connu. Attaephilus scheuerni Perreau, n'avait fait l'objet que d'une diagnose préliminaire, et nous complétons ici sa description. D'autres espèces sont nouvelles: Ptomaphaginus balazuci n. sp., Ptomaphaginus baliensis n. sp., Ptomaphaginus riedeli n. sp., et une espèce présentant un intérêt particulier, du point de vue phylogénétique et biogéographique: Choleva (Protocatops) medvedevi n. subgen., n. sp., première espèce de Choleva vietnamienne.

Le genre Choleva, essentiellement paléarctique, n'était connu de l'Asie du sudest que par une seule espèce distribuée au Népal et en Assam. La présence d'une nouvelle espèce au Vietnam, d'ailleurs apparentée à l'espèce himalayenne repousse donc encore vers le sud-est l'aire de répartition du genre Choleva, les caractères particuliers communs à ces deux espèces sont la raison de la séparation du sous-genre Protocatops n. subgen. 
Abbréviations utilisées: MHNG: Muséum d'histoire naturelle de Genève; MNHNP: Muséum National d'Histoire Naturelle de Paris; SMNS: Staatliches Museum für Naturkunde Stuttgart; CMP: collection Michel Perreau.

\section{DESCRIPTIONS}

Le genre Ptomaphaginus est un groupe très homogène dont il est souvent bien difficile de distinguer les espèces sur la seule morphologie externe. Certaines espèces présentent, exceptionnellement des particularités bien marquées constituant d'intéressants caractères distinctifs: échancrures entourées ou non de dents sur les ventrites VII et VIII du mâle ( $P$. franki Perreau, $P$. trautneri Perreau), touffe de poils à l'extrémité des élytres ( $P$. pilipennis Perreau, $P$. pilipennoides Perreau), soies particulièrement longues à la base des fémurs... Toutefois, la plupart des espèces ne peuvent être distinguées que par les caractères sexuels, édéage chez le mâle, et spermathèque chez la femelle. Même les mensurations ne donnent que de vagues indications qui ne permettent plus de reconnaître le nombre croissant d'espèces. C'est la raison pour laquelle les descriptions que nous donnons des nouvelles espèces de Ptomaphaginus sont très courtes, et n'insistent que sur les vraies particularités.

\section{Ptomaphaginus balazuci n. sp.} (MNHNP).

Holotype đ: Indonésie, Ngalam Kamang près de Tinngo, le 20-7 1979, J. Balazuc leg.

Description: Longueur: 3,2 mm. Coloration générale brun foncé, les antennes uniformément plus claires. Tout le corps recouvert d'une fine pubescence dorée.

Bord postérieur du pronotum rectiligne, non sinué.

Bord apical du ventrite VIII sinué (fig. 4).

Edéage presque rectangulaire en vue dorsale, (figs 1 et 2), segment IX représenté sur la figure 3.

Femelle inconnue.

Cette espèce se reconnaît facilement par la forme de l'édéage et la sinuosité du bord postérieur du ventrite VIII.

\section{Ptomaphaginus baliensis n. sp.}

Holotype ơ: Indonésie, Bali, Mt Batukao, 500-700 m, 28-29/10/1991, I. Löbl (MHNG). Paratypes: 1 o, même origine (MHNG), 1 ò et 2 \&, Bali, Mt Agung, Besahik audessus du temple, 31/10-1/11/1991, I. Löbl (MHNG et CMP).

Description du mâle: longueur: $2,5 \mathrm{~mm}$. Coloration générale brune, les antennes brun clair. Tout le corps est recouvert d'une fine pubescence.

Bord postérieur du pronotum légèrement sinué vers l'arrière au niveau des angles.

Ventrite VIII avec une très faible sinuosité du bord postérieur. 

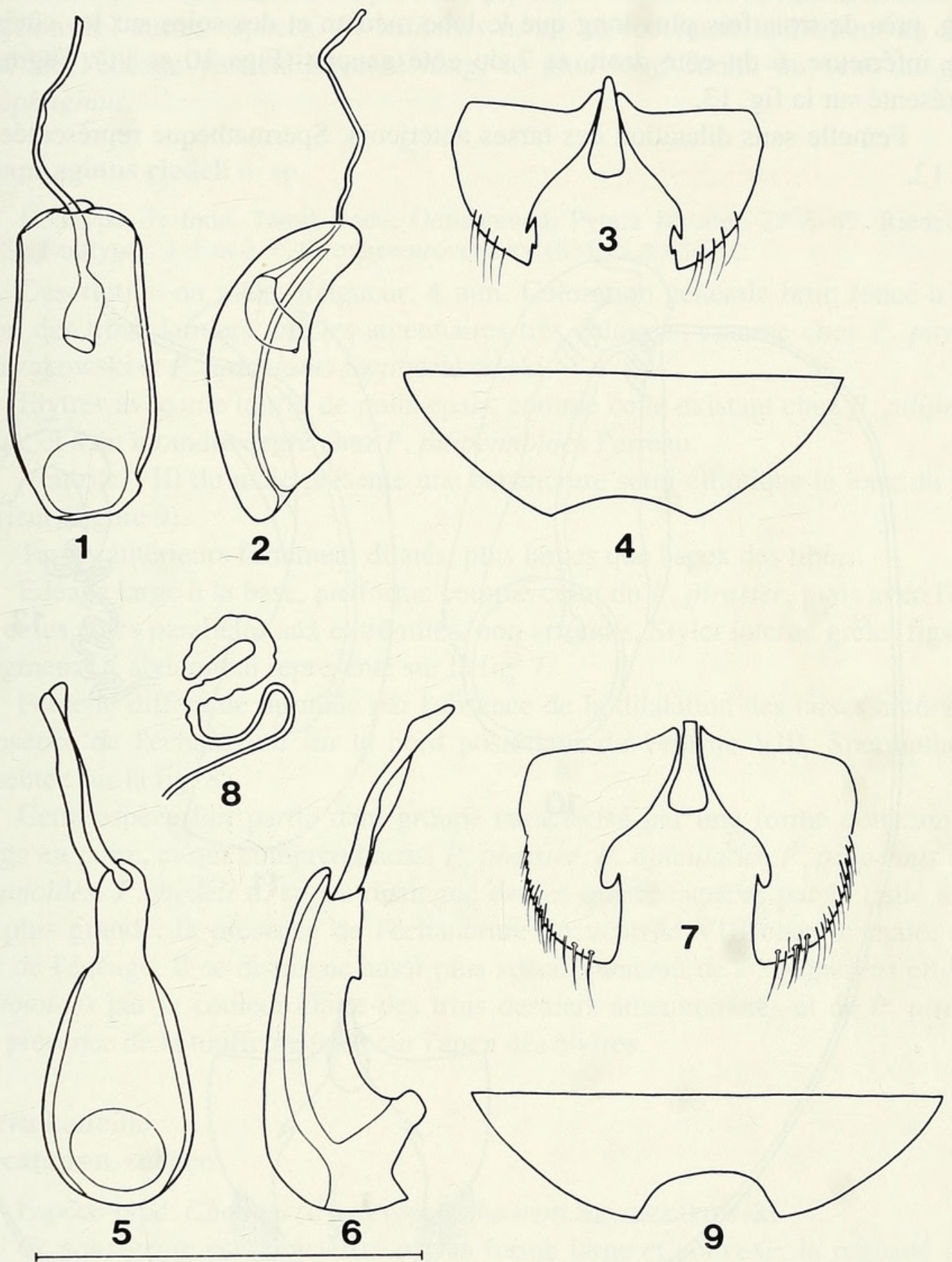

FIGS 1-9

1-4: Ptomaphaginus balazuci n. sp., holotype. 1. Edéage face dorsale; 2. Edéage face latérale; 3. Segment IX; 4. Ventrite VIII du mâle. 5-9: Ptomaphaginus riedeli $\mathrm{n}$. sp. 5. Edéage face dorsale; 6. Edéage face latérale; 7. Segment IX; 8. Ventrite VIII du mâle; 9. Spermathèque. 
Edéage particulièrement robuste, présentant un stylet interne très fin et très long, près de trois fois plus long que le lobe médian et des soies sur les côtés de la face inférieure, 6 du côté droit, et 7 du côté gauche (Figs 10 et 11). Segment IX représenté sur la fig. 13.

Femelle sans dilatation des tarses antérieurs. Spermathèque représentée sur la fig. 12.

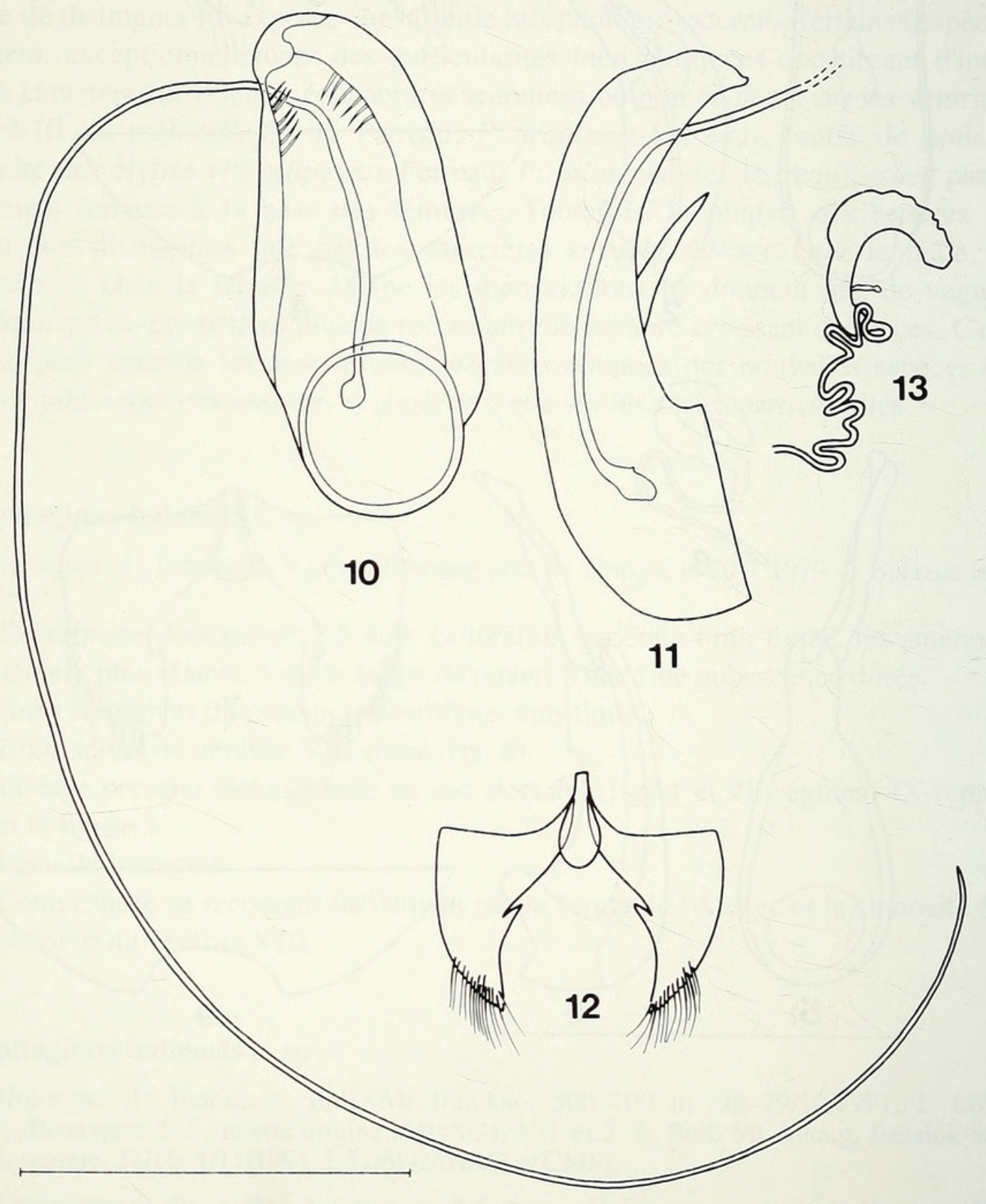

FIGS $10-13$

Ptomaphaginus baliensis n. sp. 10. Edéage face ventrale; 11. Edéage face latérale; 12. Segment IX; 13. Spermathèque. L'échelle représente $0,5 \mathrm{~mm}$. 
La morphologie externe ne présente pas d'autre caractère spécial permettant la distinction des autres espèces. $P$. baliensis n. sp. se reconnaît facilement au stylet interne de l'édéage particulièrement long, le plus long connu au sein du genre Ptomaphaginus.

\section{Ptomaphaginus riedeli $\mathrm{n}$. sp.}

Holotype o : Inde, Tamil Nadu, Ooticamund, Pytara Mysore, 22-8-89, Riedel leg. (SMNS). Paratypes: 4 ô et 3 \% de même provenance (SMNS et CMP).

Description du mâle: longueur: $4 \mathrm{~mm}$. Coloration générale brun foncé à l'exception des trois derniers articles antennaires très éclaircis, comme chez $P$. piraster Szymczakowski et $P$. apiculatus Szymczakowski.

Elytres avec une touffe de poils épais, comme celle existant chez $P$. pilipennis Perreau, et à un moindre degré chez $P$. pilipennoides Perreau.

Ventrite VIII du mâle présente une échancrure semi-elliptique le long du bord postérieur (figure 9).

Tarses antérieurs fortement dilatés, plus larges que l'apex des tibias.

Edéage large à la base, piriforme comme celui du $P$. piraster, mais avec l'apex effilé et les côtés parallèles aux extrémités, non arrondis. Stylet interne grêle (figs 5 et 6). Segment IX abdominal représenté sur la fig. 7.

Femelle différente du mâle par l'absence de la dilatation des tarses antérieurs, et l'absence de l'échancrure sur le bord postérieur du ventrite VIII. Spermathèque représentée sur la fig. 8 .

Cette espèce fait partie d'un groupe caractérisé par une forme commune de l'édéage en poire, et qui comprend aussi $P$. piraster, $P$. apiculatus, $P$. pilipennis et $P$. pilipennoides. $P$. riedeli $\mathrm{n}$. sp. se distingue de ces quatre espèces par la taille nettement plus grande, la présence de l'échancrure du ventrite VIII chez le mâle, et la forme de l'édéage. Il se distingue aussi plus spécifiquement de $P$. pilipennis et de $P$. pilipennoides par la couleur claire des trois derniers antennomères et de $P$. piraster par la présence de la touffe de poils sur l'apex des élytres.

\section{Choleva Latreille \\ Protocatops n. subgen.}

Espèce-type: Choleva (Cholevopsis) henroti Szymczakowski

Ce sous-genre est caractérisé par sa forme large et convexe, la rugosité de la ponctuation élytrale, le segment génital non sclérifié, la disparition des stries élytrales.

SzYMCZAKOWSKI (1961) décrivait Choleva (Cholevopsis) henroti récoltée en Assam. Il attribua cette espèce au sous-genre Cholevopsis à cause de la rugosité de la ponctuation élytrale, et du segment IX de la femelle non sclérifié. Il insistait toutefois sur les différences importantes existant avec les autres espèces du sous-genre. Aujourd'hui, la découverte d'une seconde espèce étroitement apparentée mais bien distincte du Vietnam nous conduit à la conclusion que $C h$. henroti n'est pas une espèce isolée, mais la première représentante d'une lignée asiatique de Choleva bien différente de toutes les autres lignées de ce genre. 
En fait, l'habitus de ces espèces rappelle étrangement celui du genre Catops:

- les stries élytrales longitudinales ont totalement disparu (sauf la suturale),

- les élytres présentent une microréticulation, plus prononcée que celle des Cholevopsis, mais n'allant pas toutefois jusqu'à la teinte cendrée des élytres des Catops,

- la forme est large et très convexe,

- la coloration générale est très foncée,

- le dernier antennomère de la Ch. medvedevi est éclairci, comme chez certains Catops.

Plusieurs caractères montrent sans ambiguïté l'appartenance aux Cholevini et non aux Catopini:

- l'édéage de la Ch. henroti présente des styles larges et épais (celui de la $C h$. medvedevi est inconnu),

- l'épistome est très nettement séparé du front par une suture clypéo-frontale très profonde,

- le ventrite VIII des femelles ne présente pas de fossette en arrière du spiculum ventrale, alors qu'il en existe une chez tous les Catopini.

Ces deux espèces constituent donc une lignée ayant conservé certains caractères ancestraux (segment IX de la femelle non sclérifié), mais présentant aussi un certain nombre d'apomorphies: absence de stries élytrales exceptée la suturale, rugosité de la ponctuation élytrale. Toutes ces raisons nous ont conduit à créer ce nouveau sous-genre.

En plus de ces caractéristiques morphologiques, cette lignée de Choleva présente une autre particularité. Les espèces semblent présenter une affinité particulière pour des zones tropicales ou subtropicales, ce qui n'est le cas d'aucune espèce des autres sous-genres.

\section{Choleva (Protocatops) medvedevi n. sp.}

Holotype \&: Vietnam, province de Vinh-Phu, Tamdao, 800-1200 m, 12/22-4-1966, leg. Medvedev, Golovatch (SMNS).

Description: longueur: 4,3 mm. Forme large et convexe. Coloration générale brun très foncé, presque noir, les 6 premiers antennomères et l'extrémité du dernier plus clairs. Tout le corps couvert d'une fine pubescence dorée dressée. Tête, pronotum et élytres entièrement et très finement microréticulés.

Antennes très élancées, les longueurs de chaque antennomère en microns $( \pm$ 10) sont les suivantes: 200, 140, 120, 130, 150, 130, 120, 120, 170, 170, 200.

Pronotum ovale, 1,8 fois plus large que long, avec tous les angles très largement arrondis, et nettement plus étroit que les élytres. Ponctuation beaucoup plus fine, mais la microréticulation semblable à celle des élytres.

Elytres très larges et très convexes, seulement 1,23 fois plus longs que larges, et 1,5 fois plus larges que les pronotum. Stries longitudinales totalement absentes 
excepté la strie suturale. La ponctuation est rugueuse entremêlée d'une fine microréticulation.

Tibias intermédiaires légèrement arqués.

Segment IX abdominal non sclérifié.

Spiculum ventrale étroit, les côtés parallèles, arrondi en avant (fig. 19).

Mâle inconnu.

Cette espèce est très semblable à Choleva henroti Szymczakowski décrite d'Assam (SzYMCZAKOWSKi 1961, 1964), et retrouvée par la suite au Népal (PERREAU, 1988). Elle s'en distingue par les caractères suivants:

- taille plus grande,

- huitième antennomère moins allongé, à peu près carré au lieu de 1,4 fois plus long que large,

- tibias postérieurs moins arqués,

- le caractère le plus important est la forme très différente du spiculum ventrale, étroit, parallèle et arrondi à l'extrémité chez Ch. medvedevi, triangulaire à base large, et anguleux à l'extrémité chez Ch. henroti (Figs 18 et 19).

\section{Choleva gabriellae Giachino}

Algérie: Atlas de Blida, Chréa sur Blida, 1400 m, 3-5-1988, Besuchet, Löbl et Burckhardt, 1 o et 1 (MHNG).

La femelle était inconnue jusqu'alors. Le ventrite VIII et le spiculum ventrale sont représentés sur la fig. 20 et le segment IX sur la fig. 21.

Lors de sa description, Giachino n'a pas tranché entre l'appartenance au groupe "cisteloides" ou au groupe "reitteri". Cette espèce présente en effet des caractères intermédiaires, la structure du sac interne de l'édéage la rapproche du groupe "cisteloides" et la forme générale du corps, la forme des trochanters postérieurs mâles et la présence de soies dressées sur les élytres la rapprochent du groupe "reitteri". Les deux groupes sont étroitement apparentés, et les caractères utilisés par Jeannel pour les séparer ne sont pas clairement des apomorphies.

\section{Attaephilus scheuerni Perreau}

Seule avait été publiée une diagnose préliminaire de cette espèce (PERREAU, 1994). Nous en donnons ici une description plus détaillée. La série typique ne se trouve plus dans la collection Scheuern comme indiqué dans la diagnose. Le matériel qui a servi à la description est le suivant: holotype $\delta$ : Paxos, Corfu (Kerkira), 2 km NW Gaios, 17-4-81, J. Scheuer (SMNS); paratype: 1 o même provenance (CMP).

Description: longueur: $3,2 \mathrm{~mm}$. Coloration générale brun-jaune clair. Tout le corps recouvert d'une double sétulation dorée, l'une composée de soies courtes et couchées, l'autre de soies longues et dressées.

Pronotum 1,8 fois plus large que long, les angles postérieurs très étroitement arrondis, la base saillante en arrière, arrondie en son milieu. Côtés régulièrement rétrécis de l'arrière vers l'avant, la plus grande largeur à la base. Soies dressées du 


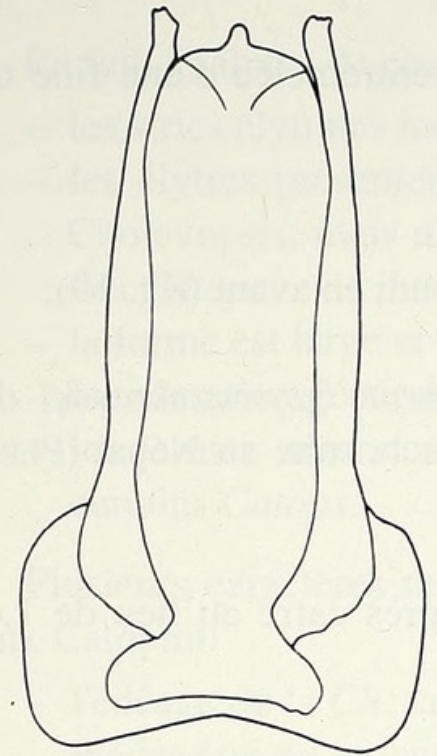

14

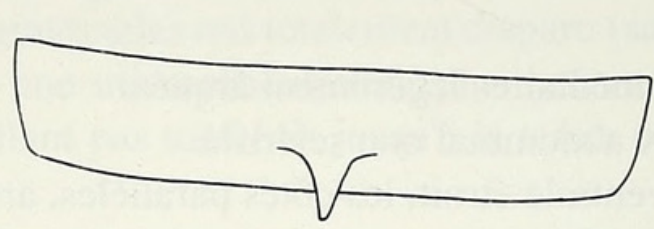

15

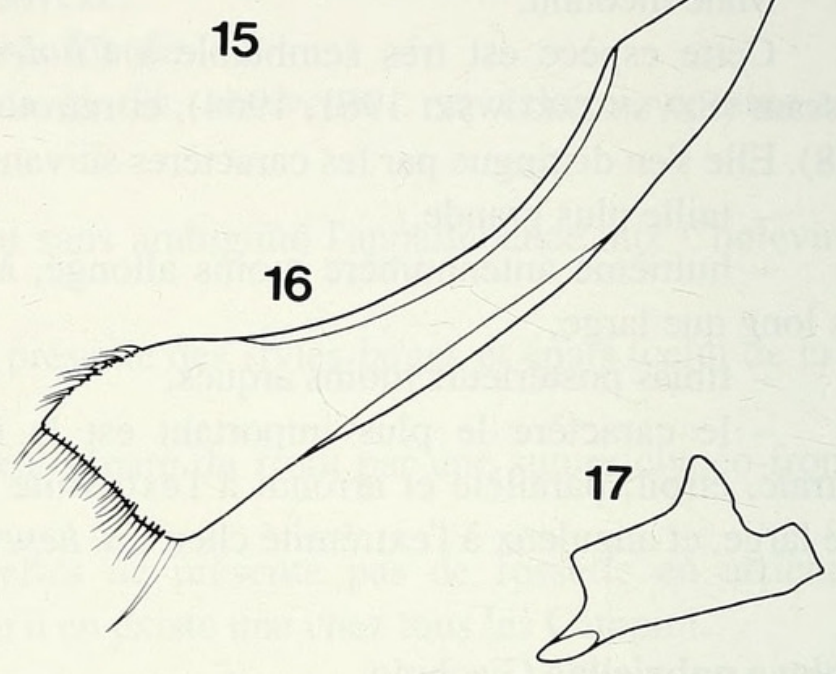

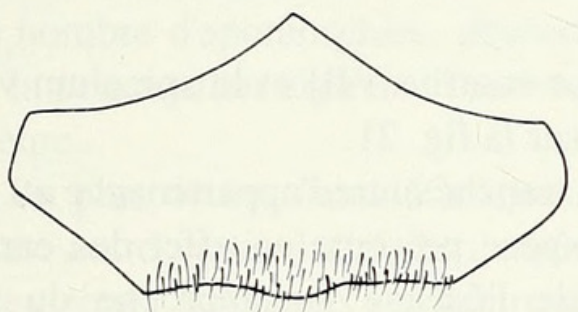

18

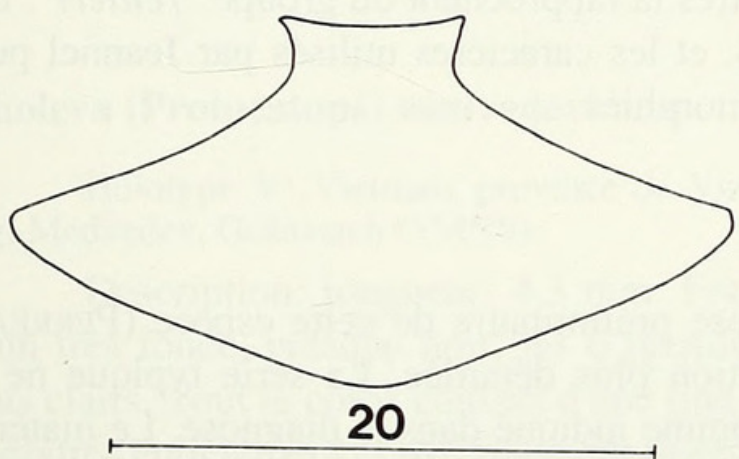

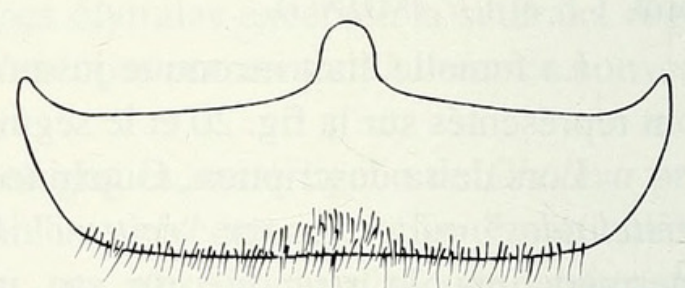

19

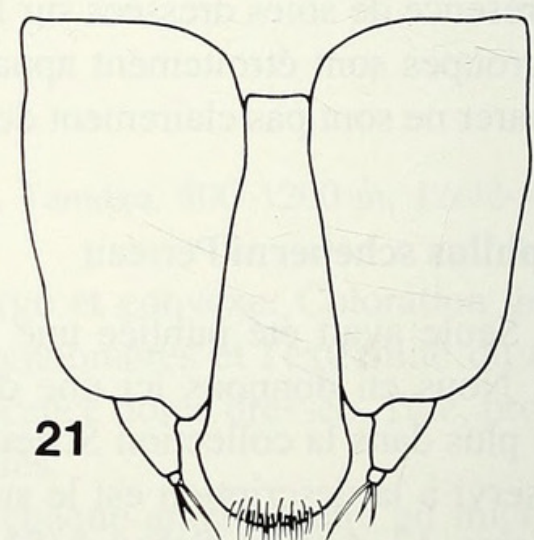

FIGS 14-21

14-18: Attaephilus scheuerni Perreau. 14. Edéage face dorsale; 15. Ventrite V du mâle; 16. Tibia intermédiaire mâle; 17. Trochanter intermédiaire droit du mâle, face ventrale. 18. Choleva (Protocatops) henroti Szymczakowski, ventrite VIII femelle et spiculum ventrale. 19: Choleva (Protocatops) medvedevi n. sp. holotype, ventrite VIII femelle et spiculum ventrale. 20 et 21: Choleva (s. str.) gabriellae Giachino. 20. ventrite VIII femelle et spiculum ventrale; 21. Segment IX face dorsale. L'échelle représente $0,5 \mathrm{~mm}$. pour les figs 14, 16, 17, 19, 20, 21, et 1 mm pour les figs $15,18,19,20,21$. 
pronotum particulièrement bien visibles à la base le long de laquelle un certain nombre d'entre elles s'alignent.

Elytres à ponctuation rugueuse et irrégulière. Pubescence dressée alignée longitudinalement.

Ventrite V avec une très forte dent médiane (fig. 15). Trochanters intermédiaires aplatis en une palette épaisse perpendiculaire au plan du corps (fig. 17).

Face interne des tibias intermédiaires très échancrée. Face supérieure ornée de deux carènes, l'une sur le bord interne s'étendant sur toute la longueur du tibia excepté une très courte distance à la base, l'autre sur le bord externe, s'étendant sur le tiers médian. Tibia représenté sur la figure 16. Tarses antérieurs peu dilatés, à peine plus larges que le tibia.

Edéage large, rétréci très brusquement à l'apex, avec un petit bouton apical. Sac interne armé de deux phanères latérales constituées de dents fines et d'une rangée centrale de dents nettement plus épaisses. Paramères épais sauf à l'apex, et divergents aux extrémités (fig. 14).

La conformation des tibias intermédiaires rapproche cette espèce de $A$. arenarius Hampe. Par contre, l'édéage ressemble à celui de A. funebris Reitter.

Le genre Attaephilus peut être divisé en trois groupes d'espèces. L'un caractérisé par un édéage effilé et pointu à l'apex, et avec des antennes courtes à massue compacte. Il comprend Attaephilus laticornis Frank \& Perreau, A. weisi Reitter, et A. servati Coiffait. Ce groupe ressemble aux Catopomorphus sauf la pubescence beaucoup plus longue. Le second groupe présente aussi un édéage du même type que le groupe précédent, mais les antennes sont plus longues et plus déliées. Il comprend Attaephilus arenarius Hampe, punctipennis Jeannel, paradoxus (Motschoulsky) et weiratheri Jeannel. Le troisième groupe est caractérisé par un édéage quadrangulaire en vision dorsale, rétréci très brusquement à l'apex, terminé ou non par un petit bouton apical. Les antennes sont également longues et grèles. Il comprend les Attaephilus funebris Reitter, rambouseki Jeannel, illyricus Jeannel, angustus (Reitter), persicus Szymczakowski, niger Karaman. Les mâles d'A. molitori Scheerpeltz et reitteri Jeannel sont inconnus et ne peuvent être situés actuellement dans ces groupes. A. scheuerni n. sp. se place dans le troisième groupe et se distingue des autres espèces par la forme des tibias intermédiaires mâles.

Les deux exemplaires connus ont été récoltés dans une fourmilière de Messor structor.

\section{REMERCIEMENTS}

Je tiens à remercier toutes les personnes qui m'ont confié l'étude du matériel figurant dans cet article ainsi que celles qui m'ont permis d'accéder aux collections pour effectuer les comparaisons nécessaires aux déterminations et aux descriptions: J. Balazuc (Nogent sur Marne), N. Berti (Muséum National d'Histoire Naturelle, Paris), I. Löbl (Muséum d'histoire naturelle de Genève), W. Schawaller (Staatliches Museum für Naturkunde, Stuttgart), J. Scheuern (Sinzig-Westum). Je remercie aussi J. 
Weulersse (Muséum National d'Histoire Naturelle, Paris), pour la détermination de la fourmi hôte de l'Attaephilus scheuerni, et I. Ruzicka (Prague) pour ses intéressantes remarques.

\section{RÉFÉRENCES}

Giachino, P.M. 1985. Choleva (s. str.) gabriellae e Sciodrepoides casalei nuove specie della Kabylia e note corologiche sui Catopidi d'Algeria (Coleoptera, Catopidae). Boll. Mus. Reg. Sc. Nat., Torino, 3: 337-348.

Perreau, M. 1988. Les Cholevidae himalayens du Muséum d'Histoire naturelle de Genève. Revue Suisse Zool., 95: 1005-1018.

Perreau, M. 1994. Diagnose préliminaire d'une nouvelle espèce d'Attaephilus de Grèce (Coléoptères, Cholevidae). Bull. Soc. ent. France, 99: 384.

SzYMCZAKOwSKI, W. 1961. Eléments paléarctiques dans la faune orientale des Catopidae (Coleoptera). Acta zool. cracov., 6: 123-136.

SzYmCZAKOWSKI, W. 1974. Nouvelles remarques sur les Catopidae (Coleoptera) de la région orientale. Acta zool. cracov., 19: 197-216.

SzYmcZaKowski, W. 1975. Catopidae (Coleoptera) récoltés dans le sud de l'Inde par l'expédition du Muséum de Genève en 1972. Acta zool. cracov., 20: 121-149. 


\section{$2 \mathrm{BHL}$ Biodiversity Heritage Library}

1995. "Especes nouvelles ou mal connues de Cholevidae (Coleoptera)." Revue suisse de zoologie 102, 969-978. https://doi.org/10.5962/bhl.part.80486.

View This Item Online: https://www.biodiversitylibrary.org/item/128625

DOI: https://doi.org/10.5962/bhl.part.80486

Permalink: https://www.biodiversitylibrary.org/partpdf/80486

\section{Holding Institution}

Smithsonian Libraries

\section{Sponsored by}

Biodiversity Heritage Library

\section{Copyright \& Reuse}

Copyright Status: In Copyright. Digitized with the permission of the rights holder

Rights Holder: Muséum d'histoire naturelle - Ville de Genève License: http://creativecommons.org/licenses/by-nc-sa/3.0/ Rights: https://www.biodiversitylibrary.org/permissions/

This document was created from content at the Biodiversity Heritage Library, the world's largest open access digital library for biodiversity literature and archives. Visit BHL at https://www.biodiversitylibrary.org. 